\title{
RAPID VISUALIZATION OF THE MASS DISTRIBUTION PROFILE OF ELECTROSPUN LAYERS
}

\author{
Marek Pokorný*, AdéLa Kotzianová, Vladimir Rassushin, \\ JAN KLEMEŠ, VLADIMÍR VELEBNÝ
}

\author{
Contipro a.s., Dolní Dobrouč 401, 561 02, Czech Republic \\ * corresponding author: marek.pokorny@contipro.com
}

\begin{abstract}
Nanofibrous materials are currently widely used in many different areas. One of the technologies commonly used for their production is electrospinning. The mass distribution in a produced nanofibrous layer is a crucial factor especially for filtration applications and active ingredients or drug release applications. Image analysis is an efficient non-destructive method of a mass distribution determination, which has already been used in the evaluation of textiles and melt-blown webs, but so far only a few publications have dealt with its use in the field of electrospun materials. To facilitate the process of image analysis of deposited nanofibers, we developed a new collector electrode, which makes it possible to determine the optical mass distribution profile of the produced layer. A series of experiments proved that the developed collector allows an easy evaluation of the appearance of nanofibrous layers as well as the effects of many associated factors such as emitter configuration on the resulting sample. The results showed how the properties of nanofibrous layers (such as deposition zone dimensions, location of these zones, mass distribution profiles produced by different spinning nozzles) can change during the electrospinning process. The advantages of the method described in the present article are that it allows a contactless and non-destructive determination of mass distribution, detection of macroscopic defects, and investigation of causes behind layer thickness inhomogeneities. All in all, the presented method is a useful research and development tool for the field of electrospinning.
\end{abstract}

KEYWORDS: nanofibrous layers; electrospinning; mass distribution profile; image analysis.

\section{INTRODUCTION}

Nanofibrous materials are currently widely used in many different areas. Nanofibrous and submicrofibrous materials draw interest due to their high surfaceto-volume ratio and the applicable properties of the input polymers. Electrostatic spinning is a versatile technology for the production of micro- and nanofibers. Nowadays, it is widely used in many fields including filtration [1], tissue engineering [2, 3], electrochemistry [4], sensors [5], composites [6], drug delivery [7], and biomedical applications [8]. Each of these applications requires specific physical and biological properties of the produced layer, which are heavily dependent on solution properties and numerous process variables [9].

A basic electrospinning setup includes a spinning electrode, which is connected to a high-voltage power source, and a collector electrode. The collector is usually a grounded flat plate, drum, etc. 99, commonly made of a non-transparent electrically conductive material. Furthermore, fibres are, in most cases, spun onto various substrates, such as aluminium foils and nonwoven fabrics. Both the non-transparent nature of the collectors and the use of substrates prevent a direct assessment of the spinning process and its results. However, there are many parameters that affect the electrospinning process and, therefore, fibre deposition such as electrospinning is a highly multi- variable method (electrode arrangement, electrostatic field formation, solution properties, atmospheric conditions, etc.) 9 12. A research on electrospinning causalities includes measurements of various material properties including mechanical, chemical, and biophysical, as well as basic layer properties, such as fiber quality and distribution. The most basic and at the same time the most important parameter the morphology of the layers - is usually evaluated using only SEM images, and characteristics of the entire layer are assessed very rarely. However, to precisely determine parameters like mechanical durability or drug release, it is necessary to analyse layers in their entirety and identify or rule out any defects and irregularities (e.g., in thickness). One possibility is to determine the mass distribution within the layers, which provides information about the real shape and size, and about displacement of the deposition zone. Furthermore, one of the crucial factors for many applications - uniformity of thickness distribution - can also be determined from these data. Several papers have already been published on the topic of analysis of the electrospinning process and the resulting layers [13, 14. These papers deal both with the theory, e.g., the shaping of the electrostatic field during the electrospinning process and its effects on the prepared nanofibrous layer, and with direct analyses of prepared layers. Nevertheless, the nanofibrous layers studied in these papers were analysed mostly on 
A)

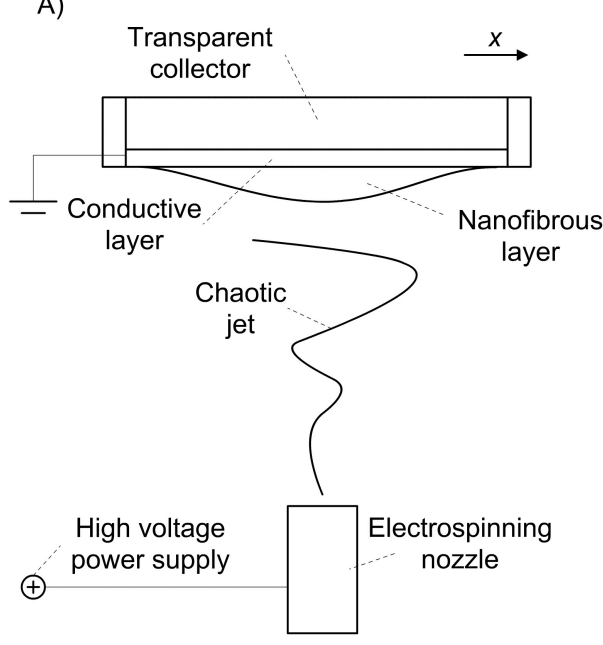

B)

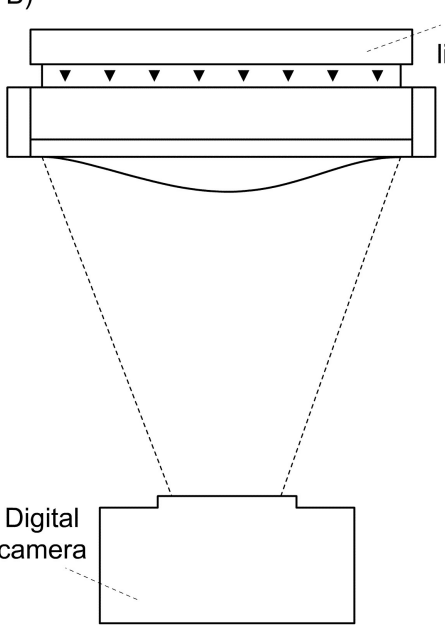

C)

Diffusing

light source
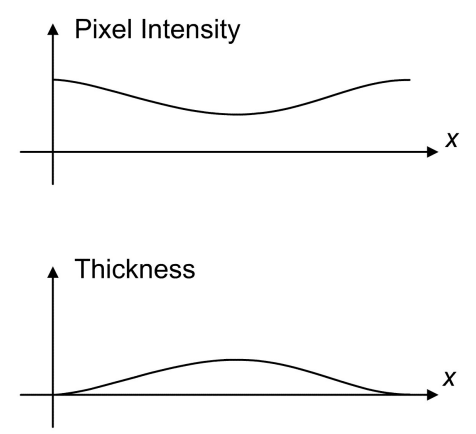

$f_{(\text {Pixel Intensity) }}=f_{(\text {Thickness })^{-1}}$

FigURE 1. Diagrams illustrating the deposition of nanofibers onto the transparent collector (A), and the use of a LED source and a digital camera to image the resulting layer with the help of transmitted light (B). The pixel intensity profile of the acquired image corresponds to the thickness profile of the layer (C).

non-transparent substrates, such as aluminium foil, or using merely a point-based analysis. Beisel et al. [15] focused on a theoretical determination of the area of an electrospun layer in dependence on the intensity of the electrostatic field and the magnitude of electrostatic forces in the vicinity of the collector. The authors compared their theoretical results with experimental results. The nanofibrous layers were spun from polyvinyl alcohol and analysed on an aluminium foil substrate. However, the paper fails to provide any information on the imaging process used to image the nanofibrous layers as well as on the methods used to process and analyse the acquired images. Lee at al. 16] used transmitted light to study the layer uniformity. The authors focused on the preparation of nanofibrous layers with high thickness homogeneity. A glass collector was used for the deposition; however, the nanofibrous layers were transilluminated at only three points, specifically at the edges and in the centre of each layer. The applied method, therefore, fails to provide comprehensive information on the distribution of thickness throughout the entire layer. Another paper dealing with the determination of thickness homogeneity was published by Zhang et al. [17] who focused on the preparation of nanofibrous filters, the quality of which is greatly dependent on the distribution of thickness throughout the layer. The authors used an optical profilometer and their samples were analysed on a glass slide. Again, the applied method failed to analyse the samples in their entirety as the measurements were point-based. Finally, Pokorný et al [18] evaluated planar samples of nanofibrous layers using transmitted light. The authors studied the effects of the movement of electrodes on the resulting homogeneity of large-area nanofibrous layers; the causes of inhomogeneities occurring in layers produced with static electrodes were not investigated/described.

In the present paper, we describe a transparent collector, which was developed to provide a very flat and transparent surface for a direct deposition of nanofibers. The collector enables a layer analysis using transmitted light and a digital camera. The gathered data are processed with an image analysis software and the results are used to evaluate both the geometric properties and the mass distribution profile of the layer. The integration of image analysis into the research and development of the electrospinning process resulted in the creation of a useful cross-functional tool.

\section{Materials and Methods}

\subsection{MATERIALS}

A $16 \%$ aqueous solution of poly(vinyl) alcohol (PVA, Fichema) was diluted to $12 \% \mathrm{w} / \mathrm{w}$ with distilled water. The solution was stirred at a room temperature for 12 hours.

\subsection{Electrospinning}

Nanofibrous samples were prepared with the 4SPIN® LAB device (Contipro a.s.) [19] at a temperature of 24$26^{\circ} \mathrm{C}$ and relative humidity of $12-23 \%$. Each sample was spun over 30 minutes. The solution was dosed through various emitters with one, two or six needles arranged in a line (17-gauge, Hamilton) and with two needleless emitters (linear and circular). The PVA solution was dosed via an inboard syringe pump at feed rates ranging from 10 to $160 \mu \mathrm{l} / \mathrm{min}$ depending on the type of the nozzle used. High voltage ranged from 10 to $58 \mathrm{kV}$ depending on the number of needles and emitter type.

The transparent collector was designed to allow the acquisition of images of deposited layers using transmitted light (see diagram in Fig. 1a). A transparent glass sheet of a size $20 \times 20 \mathrm{~cm}^{2}$ was fixed in a plastic frame. The glass was covered with an Indium Tin Oxide (ITO) coating (provided by Toptec) to make 


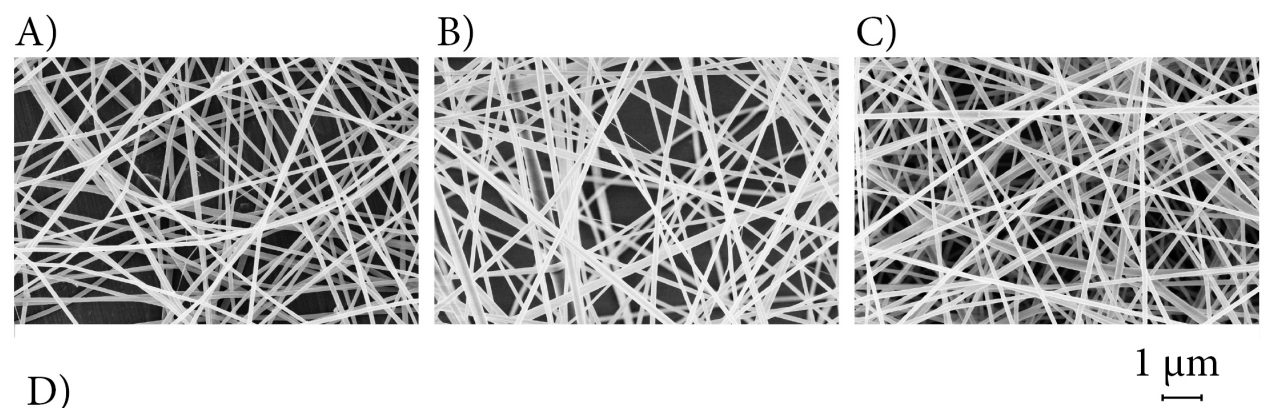

D)

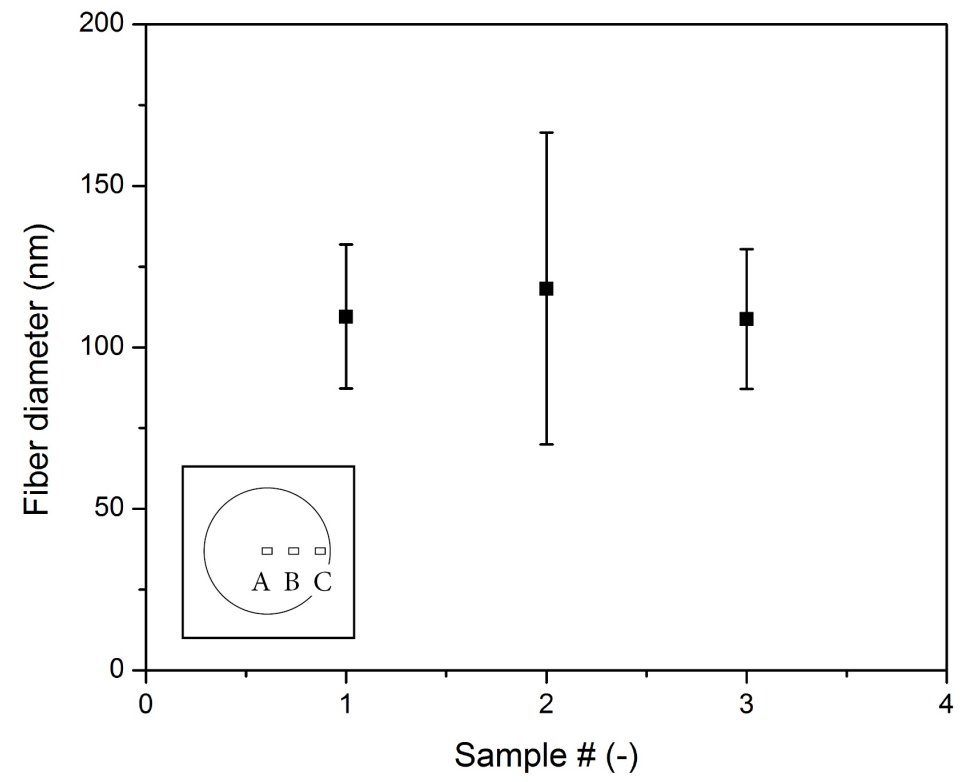

FiguRE 2. Morphology of the analyzed layer's nanofibrous structure at the center (A), at the edge (C), and in the middle between these two locations (B). The diagram (D) indicates the average fiber diameters at the analyzed locations.

it conductive and thus suitable for electrospinning as a grounded target. After each spinning process, the glass collector with the deposited nanofibrous layer was photographed in transmitted light.

\subsection{SAMPLE ANALYSIS}

The image analysis was used to assess the fibre mass distribution throughout the deposited layers as well as their basic properties. Images were taken in transmitted light (Fig. 1p). A LED panel (Metaphase Lighting Technologies) with a light intensity homogeneity of $\pm 5 \%$ was used as the light source. The dimension of the panel panel were $22 \times 40 \mathrm{~cm}^{2}$. The glass collector holding the electrospun sample was placed directly on the LED panel without any intermediate layer. Images of the samples were taken with a steady compact camera (Nikon Coolpix P7700) fixed on a tripod at a distance of $30 \mathrm{~cm}$ from the surface of the LED panel. The images were taken in a dark room where the LED panel was the only source of light. To ensure every sample was photographed under identical conditions, we set the camera to a manual mode with the following settings: 80 ISO, exposure time $1 / 160 \mathrm{sec}, \mathrm{f}-$ number 8.0. These conditions ensured that the images were sufficiently illuminated to allow their subsequent processing. The acquired images were analysed using the Image J software. Each image was converted to 32-bit grayscale (image contrast increased after conversion). The evaluation of the resulting images was based on the assumption that the thickness of the imaged nanofibrous layer is inversely proportional to the intensity of the profile obtained from its image (shown in Fig. 17). Furthermore, we assumed that errors produced by potential uneven lighting of the layer, optical defects in the lens, non- linearity of conversion of optical information to digital data, etc. had a negligible impact on the conclusions based on the results presented in this paper.

\section{Results And Discussion}

First, images for a SEM analysis were taken from three locations - at the centre of the deposit, on one of its edges, and in the middle between these two points. The measurements showed similar density and a lack of orientation patterns of the deposited fibres. The average fibre diameter was $(110 \pm 25) \mathrm{nm}$ for each of the analysed spots, see Fig. 2 Differences in the amount of light transmitted through the layers were, therefore, 

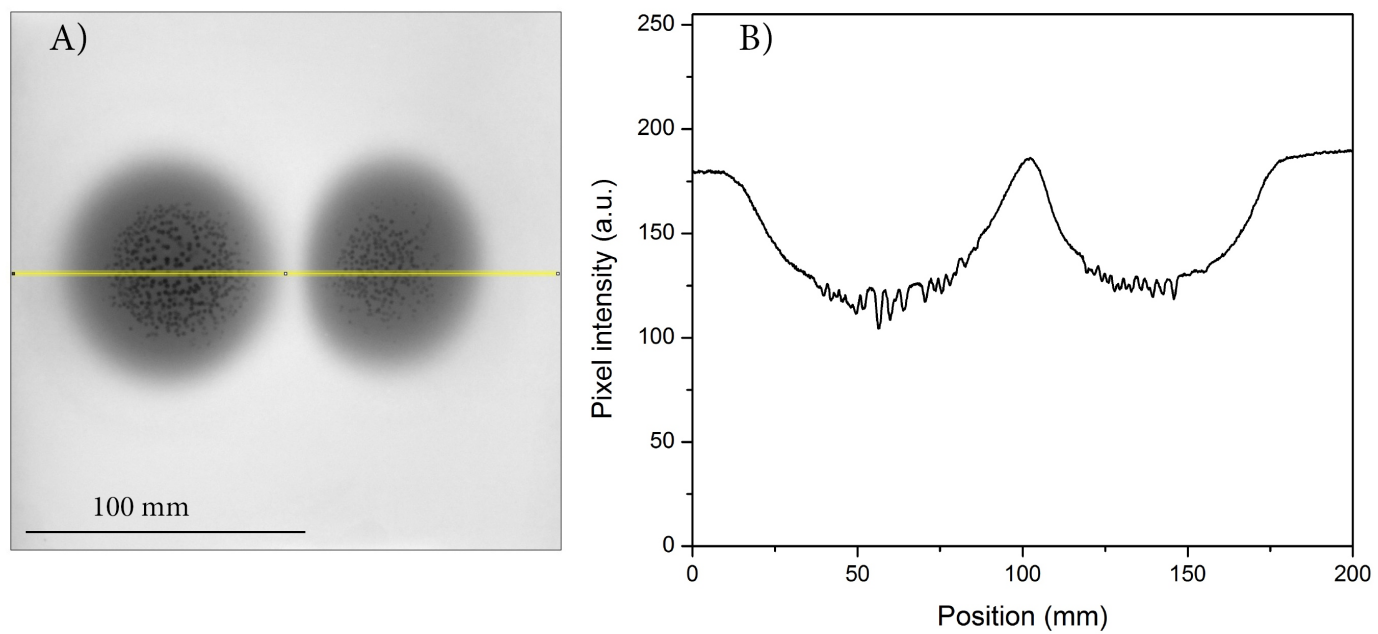

FiguRE 4. Defects in the form of local fiber clusters revealed in the nanofibrous layer (A) which appear as circular spots in the photographed image and as oscillations on the curve of the intensity profile (B) obtained from the sample along the indicated yellow line.

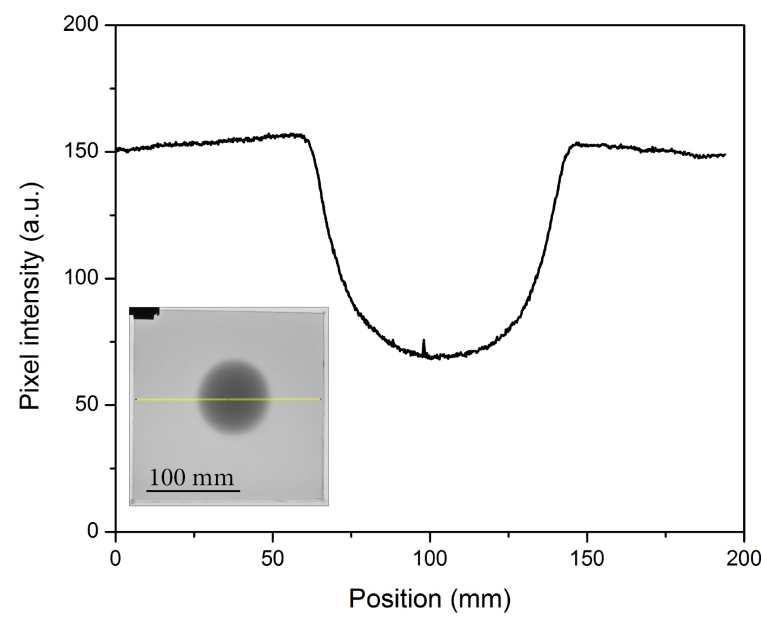

FIGURE 3. Intensity profile of pixels along the line depicted on the photograph of the transparent collector plotted as a graph. The imaged nanofibrous layer was deposited on the transparent collector using a single-needle nozzle and a basic point-to-plate configuration.

related only to the mass distribution, because no variations in fibre properties or layer structure were detected.

In the second experimental series, fibres were deposited onto the surface of the glass collector by a point-to-plate deposition. Pixel intensities measured along a straight line had a gauss-like profile, as expected (see Fig. 3). This means that the greatest amount of fibres was deposited in the centre of the deposition zone and the amounts decreased towards the sides.

The developed glass collector allowed us to directly photograph the nanofibrous layer and conduct its substrate-less analysis. This basic configuration makes it possible to study, with great accuracy, the effects of process parameters on the size and shape of the deposited layer. These process parameters include electrode distance and geometry, intensity of the applied high voltage, electric conductivity of the solution, surrounding humidity, etc. One of the advantages is that the analytical process is not affected by the properties of any substrate material. Another major advantage is the non-destructive nature of this optical method of analysis.

Light passing through a deposited layer efficiently highlights details that may be overlooked when nanofibers deposited on a nontransparent substrate are studied. However, these details fundamentally affect the quality of the produced layer and its behaviour in specific applications. For example, Fig. 4 shows clusters of $1.5 \pm 0.3 \mathrm{~mm}$ and $1.2 \pm 0.1 \mathrm{~mm}$ in diameter revealed by transmitted light in a layer deposited with two needles. Their presence is clearly shown by the pixel intensity profile where these local defects produced marked oscillations in the lowest intensity sections.

Figure 5 shows several images of nanofibrous layers with different areal mass distribution deposited with spinning nozzles of different designs. Even the photographs by themselves clearly show that the fibre distribution on the collector depends on the nozzle type used; i.e., of all the parameters, it is the design of the spinning nozzle that has the greatest effect on the thickness profile of the deposited layer. The obtained profiles of layers deposited with needle nozzles (see Fig. 5ab) demonstrate that the positively charged jets of the spinning solution repel each other on their way to the collector, resulting in the formation of thicker deposits, corresponding to the individual spinning needles, separated by areas of lesser thickness. Needleless electrodes (see Fig. 5ed) do not produce such areas of different deposition thickness, probably due to the fact that fibres are ejected by these nozzles from ran- 

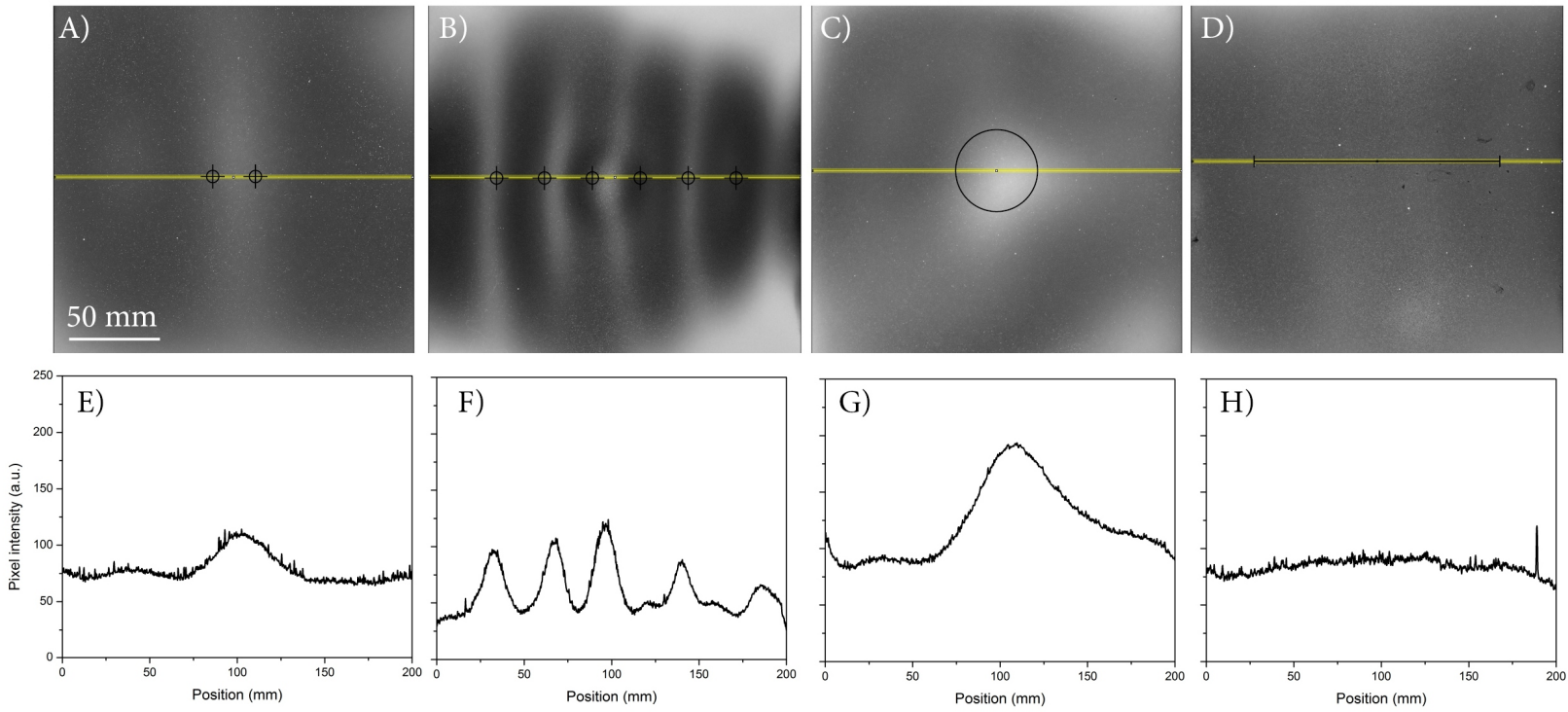

FIGURE 5. Distribution of nanofibers on the transparent collector achieved with different spinning nozzles (upper row of images A to D) and the corresponding intensity profiles of pixels along the indicated lines (lower row of images E to $\mathrm{H}$ ). The depicted layers were deposited with the following nozzles: two needles $22 \mathrm{~mm}$ apart (A and E); six needles, each two needles $25 \mathrm{~mm}$ apart (B and F); multi-jet needleless circular nozzle and electrode diameter of 44 mm (C and $\mathrm{G})$; linear needleless nozzle electrode length of $128 \mathrm{~mm}$ (D and $\mathrm{H}$ ).

domly varying places; this results in the formation of layers of a more even thickness. The nanofibers deposited in the layer shown in Fig. 5 d cover the entire surface of the collector almost completely evenly; deviations from the average pixel intensity of the image were lower than \pm 7 shades of grey, i.e., lower than $\pm 3 \%$. Our results indicate that the greatest thickness homogeneity of layers deposited on the developed glass-sheet collector can be achieved by using a linear needleless spinning nozzle.

\section{Conclusion}

A transparent plate collector for a rapid visualization of variations in mass was developed, tested, and described. The collector allows a substrate-less deposition of nanofibrous layers as well as subsequent nondestructive analysis of the properties of the deposited layers. It was shown that a combination of a simple and inexpensive image-taking setup, consisting of a LED panel and a digital camera, and the developed collector, forms a useful tool for a basic research of the electrospinning process. Several presented examples prove that the method is suitable for the visualization and potential detection of macroscopic defects in nanofibrous layers, which it allows to be imaged in the entirety of their deposition area on the collector in a single process. This cannot be done in a non-destructive way when using standard, nontransparent substrates or collectors. This newly developed imaging method allows to observe the distribution of mass in nanofibrous layers directly on the collector. The obtained results can then be used in the selection or design of spinning nozzles suitable for specific intended applications of electrospun nanofibrous layers. The examples presented in this paper demonstrate that of all the tested nozzles, the linear needleless nozzle was able to cover the collector with nanofibers most evenly. The techniques described above, used for the first time in the field of electrostatic spinning, are suitable for studying the formation of nanofibrous layers produced by various setups and electrodes both during and after the spinning process. The method is also suitable for a non-destructive evaluation of the quality of the prepared materials.

\section{REFERENCES}

[1] J. Matulevicius, L. Kliucininkas, T. Prasauskas, D. Buivydiene, D. Martuzevicius. The comparative study of aerosol filtration by electrospun polyamide, polyvinyl acetate, polyacrylonitrile and cellulose acetate nanofiber media. Journal of Aerosol Science 92:27-37, 2016. DOI: 10.1016/j.jaerosci.2015.10.006

[2] A. Capulli, L. Macqueen, S. P. Sheehy, K. Parker. Fibrous scaffolds for building hearts and heart parts. Advanced Drug Delivery Reviews 96:83-102, 2016. DOI:10.1016/j.addr.2015.11.020

[3] S. Hinderer, S. L. Layland, K. Schenke- Layland. ECM and ECM-like materials - Biomaterials for applications in regenerative medicine and cancer therapy. Advanced Drug Delivery Reviews 97:260-269, 2016. DOI:10.1016/j.addr.2015.11.019

[4] W. Luo, F. Shen, C. Bommier, H. Zhu, X. Ji, L. Hu. Na-Ion Battery Anodes: Materials and

Electrochemistry. Accounts of Chemical Research 49:231-240, 2016. DOI:10.1021/acs.accounts.5b00482 
[5] S. Lee, A. Reuveny, J. Reeder, S. Lee, H. Jin, Q. Liu, T. Yokota, T. Sekitani, T. Isoyama, Y. Abe, Z. Suo, T. Someya. A transparent bending- insensitive pressure sensor. Nature Nanotechnology 11:472-478, 2016. DOI:2016. 10.1038/nnano.2015.324

[6] G. Wang, D. Yu, A. D. Kelkar, L. Zhang. Electrospun nanofiber: Emerging reinforcing filler in polymer matrix composite materials. Progress in Polymer Science, 2017, In Press, 2017. DOI:10.1016/j.progpolymsci.2017.08.002

[7] R. Goyal, L. K. Marci, H. M. Kaplan, J. Kohn. Nanoparticles and nanofibers for topical drug delivery. Journal of Controlled Release 240:77-92, 2016. DOI:10.1016/j.jconrel.2015.10.049

[8] A. Eatemadi, H. Daraee, N. Zarghami, H. M. Yar, A. Akbarzadeh. Nanofiber: Synthesis and biomedical applications. Artificial Cells, Nanomedicine, and Biotechnology 44:111-121, 2014. DOI:10.3109/21691401.2014.922568

[9] W. E. Teo, R. Inai, S. Ramakrishna. Technological advances in electrospinning of nanofibers. Science and Technology of Advanced Materials 12:013002, 2011. DOI:10.1088/1468-6996/12/1/013002

[10] J. Deitzel, J. Kleinmeyer, D. Harris, N. B. Tan. The effect of processing variables on the morphology of electrospun nanofibers and textiles. Polymer 42:261-272, 2001. DOI:10.1016/s0032-3861(00)00250-0

[11] S. Theron, E. Zussman, A. Yarin. Experimental investigation of the governing parameters in the electrospinning of polymer solutions. Polymer 45:2017-2030, 2004. DOI:10.1016/j.polymer.2004.01.024

[12] D. Kolbuk. Tailoring of Architecture and Intrinsic Structure of Electrospun Nanofibers by Process Parameters for Tissue Engineering Applications. Nanofiber Research - Reaching New Heights, InTech, 2016. DOI:10.5772/64177, Available from: https :

//www. intechopen.com/books/nanofiber-researchreaching-new-heights/tailoring-of-architectureand-intrinsic-structure-of-electrospunnanofibers-by-process-parameters-fo
[13] G. Campatelli, L. Sapuppo, A. Scippa, Analytical FE simulation of a multi-jet electrospinning process to predict material flow. Simulation Modelling Practice and Theory, 52:135-148, 2015. DOI:10.1016/j.simpat.2014.12.005

[14] C. J. Angammana and S. H. Jayaram, The Effects of Electric Field on the Multijet Electrospinning Process and Fiber Morphology, IEEE Transactions on Industry Applications 47:1028-1035, 2011. DOI:10.1109/TIA.2010.2103392

[15] J. D. Beisel, J. Kyeremateng, L. Purkett, J. M. Andriolo, J. L. Skinner. Analytical parametric model used to study the influence of electrostatic force on surface coverage during electrospinning of polymer fibers. Journal of Vacuum Science E Technology B, Nanotechnology and Microelectronics: Materials, Processing, Measurement, and Phenomena 32, 2014. DOI:10.1116/1.4900608

[16] J. Lee, Y. H. Jeong, D. W. Cho. Fabrication of Nanofibrous Mats with Uniform Thickness and Fiber Density. Macromolecular Materials and Engineering 299:1052-1061, 2014. DOI:10.1002/mame.201300390.

[17] Q. Zhang, J. Welch, H. Park, C. Y. Wu, W. Sigmund, J. C. Marijnissen. Improvement in nanofiber filtration by multiple thin layers of nanofiber mats. Journal of Aerosol Science 41:230-236, 2010. DOI:10.1016/j.jaerosci.2009.10.001

[18] M. Pokorný, J. Klemeš, A. Kotzianová, T. Kohoutek, V. Velebný. Increased thickness uniformity of large-area nanofibrous layers by electrodynamic spinning. AIP Advances 7:105214, 2017. DOI:10.1063/1.4998489

[19] M. Pokorný, J. Novák, J. Řebíček, J. Klemeš, V. Velebný. An Electrostatic Spinning Technology with Improved Functionality for the Manufacture of Nanomaterials from Solutions. Nanomaterials and Nanotechnology 5:17, 2015. DOI:10.5772/60773 